\title{
Title: Competitive dynamics underlie cognitive improvements during sleep
}

Authors: Pin-Chun Chen ${ }^{1,2}$, Hamid Niknazar ${ }^{1}$, William A. Alaynick ${ }^{3}$, Lauren N. Whitehurst ${ }^{4}$, and Sara C. Mednick ${ }^{1 *}$

\author{
Affiliations: \\ ${ }^{1}$ Department of Cognitive Sciences, University of California, Irvine \\ ${ }^{2}$ Department of Statistics, University of California, Irvine \\ ${ }^{3}$ ScholarNexus, LLC \\ ${ }^{4}$ Department of Psychology, University of Kentucky \\ *Corresponding author. Email: mednicks@uci.edu
}

\begin{abstract}
A central debate in cognitive neuroscience is whether working memory (WM) and long-term memory (LTM) are served by separate or overlapping systems. Both memory domains rely on offline periods that include sleep to facilitate performance improvement; however, the precise neural mechanisms are not known. Prior animal studies suggest that offline neural activity supporting WM and LTM may demonstrate mutual antagonism; but this possibility and its functional significance has not been studied in humans. Using pharmacological intervention and computational modeling, we demonstrate a trade-off between neural mechanisms supporting LTM and WM processing with functional significance for performance. The findings suggest that sleep is a competitive arena in which neural mechanisms supporting LTM and WM vie for resources.
\end{abstract}

One-Sentence Summary: Sleep is a competitive arena in which neural mechanisms supporting long-term memory and working memory vie for resources. 


\section{Main Text:}

Working memory (WM) and long-term memory (LTM) serve separate functions and the idea that they are supported by separate systems has become a core assumption of modern cognitive psychology [1]. WM is a control process for planning and carrying out behavior that is information-independent, whereas LTM is an information-dependent vast store of knowledge and record of prior events. Anatomically, the memory domains appear to rely on distinct neural substrates, the prefrontal cortex (PFC) [2] and hippocampus [3], respectively. Yet, recent results have shown overlap in brain regions activated during tasks probing LTM and WM, interdependence in performance on WM and LTM tasks [4], and subtle but noticeable long-term memory deficits in patients with prefrontal brain lesions [5]. These findings are consistent with the idea that working and long-term memory may be part of a single system that can functionally differentiate information storage for different goals [6]. But the identification of neural mechanisms that support either the multi- or single-system model are lacking, underscoring the need to determine shared and distinct mechanisms underlying these two types of memory.

Both WM and LTM rely on offline periods that include sleep to facilitate performance improvement. According to the framework of systems consolidation, long-term memories are initially processed by a fast learning system in the hippocampus that binds information into transient representations (i.e. encoding). With repeated reactivation, the memories eventually become less reliant on the hippocampus and more stabilized in long-term cortical stores (i.e., consolidation). Converging evidence suggests that sleep may be an optimal offline period for consolidation as it facilitates dialogue between the hippocampus and the neocortex [7]. Specifically, during non-rapid-eye-movement (NREM) sleep, thalamic sigma oscillations, nested in cortical slow oscillation (SO) up-states, group hippocampal sharp wave ripples (SPW-R) and reactivate memory traces, suggesting sigma-mediated information transfer between hippocampal and neocortical cell assemblies [8,9]. Experimental interventions that boost sigma in humans enhance hippocampal long-term memory [10], implicating sigma as an indicator of systems consolidation.

Classic models of WM propose two governing mechanisms: 1) an active maintenance of information online through the elevated firing of prefrontal neurons, and 2) a supervisory executive control process that is supported by a prefrontal-subcortical inhibitory network $[2,11]$. This circuitry innervates the heart via sympathetic stellate ganglia and parasympathetic vagal nerve efferents. As such, cardiac autonomic activity is thought to reflect effective functioning of prefrontal control processing [12]. Accordingly, vagally-mediated heart rate variability (RMSSD and HF HRV) during wake correlates with executive function tasks that rely on PFC activity, including WM [13]. Recent studies have shown that WM improvement only occurs when the interval between training sessions contains a period of sleep [14]. Given that NREM sleep is a period of vagal dominance compared with wake, a recent study identified vagal HF HRV during NREM as a strong predictor of WM improvement [15].

Together, theoretical models and empirical data suggest that NREM sleep may support improvement in WM via strengthening of prefrontal-autonomic inhibitory networks, as well as the formation of LTM via thalamocortical sigma activity. The question is how the sleeping brain performs both of these complex feats and which sleep features are associated with these processes? Prior research suggests a potential antagonistic relation between the two neural processes during NREM sleep. Using ripple-triggered fMRI in monkeys, Logothetis and colleagues demonstrated that ripples orchestrate a privileged state of enhanced central brain activity by silencing output from the diencephalon, midbrain and brainstem, regions associated 
with autonomic regulation, which may serve to boost communication between hippocampus and cortex [16]. On the other hand, stimulating the locus coeruleus (LC), an area thought to mediate propagation of vagal nerve activity to higher-order neural regions, blocks the generation of ripple-associated sigma activity and causes hippocampal-dependent spatial memory deficits [17]. These studies suggest a potentially antagonistic interplay between sigma-dependent and vagaldependent processing. However, this possibility and its functional significance has not been studied in humans. Evidence and characterization of this relation would inform fundamental questions about the shared or distinct mechanisms of working and long-term memory.

In the present study, we enacted a pharmacological strategy to investigate the bidirectional interplay between central sigma-dependent and autonomic vagal-dependent processing during overnight sleep and its impact on LTM and WM. To address the potential antagonistic link between sigma and vagal activity, we pharmacologically increased sigma activity during overnight sleep using a GABA-A agonist, zolpidem, and measured changes in vagal activity, sigma-vagal interaction, and long-term vs working memory performances. We computationally tested our model that central sigma activity would suppress autonomic vagal activity using effective connectivity [18]. We hypothesized that 1) zolpidem would enhance sigma activity during NREM sleep, 2) zolpidem would suppress vagal activity during sleep, 3) sigma increases would boost information flow exerted from central over autonomic regions, 4) sigma-guided vagal suppression would result in parallel behavioral effects with greater long-term memory and worse working memory, and 5) the magnitude of central over autonomic information flow would directly predict the behavioral trade-off between long-term and working memory.

By exploiting the amplification of sigma during NREM sleep, we identified a novel antagonistic relationship between central and autonomic activity during sleep. Furthermore, the degree of mutual antagonism between central and autonomic activity predicted a heretofore unreported behavioral trade-off between long-term memory and working memory. These results suggest that NREM sleep confers benefits to working and long-term memory by switching between separate offline mechanisms, i.e., vagally-mediated and sigma-meditated processes. Furthermore, this sleep switch can be biased towards long-term memory consolidation by increasing sigma activity, in this case pharmacologically, and presumably by other methods as well. These results illuminate the shared and distinct fundamental mechanisms underlying longterm and working memory processes

\section{$\underline{\text { Results }}$}

\section{Experiment 1 .}

Based on previous findings, we predicted that central sigma power would have an inhibitory effect on cardiac vagal tone. To this end, we administered zolpidem in a double-blind, placebo-controlled, randomized cross-over design, in which each participant experienced two nights per drug condition (zolpidem or placebo; a total of 4 nights; $M_{\text {age }}=20.88 \pm 1.88$ years, 17 Females), with EEG and ECG monitored (Figure 1 shaded area). The order of drug conditions was counterbalanced with at least a one-week interval between the experimental visits to allow for drug clearance. We performed power spectral analysis to quantify normalized sigma activity and analyzed HRV profiles. Our intervention was successful, whereby zolpidem enhanced sigma activity during stage 2 sleep (central channels: $\mathrm{t}=2.112, \mathrm{p}=.0349$; parietal channels: $\mathrm{t}=2.214$, 
$\mathrm{p}=.0270$, corrected by Tukey's multiple comparisons; Supplemental Table S6), consistent with prior literature.

As we hypothesized, zolpidem not only increased sigma activity, but also selectively suppressed vagal tone during sleep, measured by RMSSD (Figure 2a) and high-frequency HRV (HF; Figure 2b), but had no impact on low-frequency HRV (LF; Figure 2c). Other HRV indices were reported in Supplemental Table S4.

We then tested our hypothesis that central sigma power would exert greater causal influence over vagal autonomic activity and such difference would be increased by zolpidem. To test this prediction, we used effective connectivity estimation (Figure 3a). In particular, we tested the hypotheses that central sigma naturally exercises greater causal influence on autonomic vagal activity in the placebo condition, and that increasing sigma with zolpidem would increase causal information flow from sigma to vagal activity, while decreasing the causal information flow from vagal to sigma activity in the zolpidem condition. For each subject, we calculated two measures: HFOutflow and HFInflow (see Methods). We confirmed our hypothesis that central sigma power exerted greater flow on vagal activity in the placebo condition $(\mathrm{p}<.0001$; Figure $3 b)$. We also confirmed that such difference was increased by zolpidem $(\mathrm{p}=.0369$; Figure $3 b)$. Next, we calculated a composite score, the effective connectivity ratio: HFInflow over HFOutflow, where higher numbers represented greater central sigma control over autonomic vagal activity. We observed a higher effective connectivity ratio during the zolpidem night (p $=.0059)$. Taken together, Experiment 1 verified our hypotheses that central activity naturally exerts dominance over autonomic activity during NREM sleep, and that increasing sigma activity via zolpidem inhibits vagal activity and enhances central sigma control over autonomic vagal activity.

\section{Experiment 2.}

In an independent sample of participants $\left(N=38 ; M_{\mathrm{age}}=20.85 \pm 2.97\right.$ years; 19 Females $)$, we added a behavioral experiment (Experiment 2; Figure 1) to the original design of Experiment 1 to test whether we could replicate the physiological results of Experiment 1 and determine their functional importance for sleep-dependent cognition. Again, we exploited zolpidem to modulate the interaction between central sigma and autonomic vagal activity, and examined its impacts on the improvements of LTM and WM (Figure 1). The order of drug conditions was counterbalanced with at least a one-week interval between the two experimental visits to allow for drug clearance. Due to the pharmacodynamics of zolpidem, which has a half-life of (1.5-4.5 h), and onset (mean Tmax 1.6h [19]), we divided the night into quartiles and focused our analyses on quartile two and three combined in order to maximize differences in drug conditions. We hypothesized that sigma-guided vagal suppression effects would result in parallel behavioral effects with greater long-term memory and reduce improvement in working memory. We further hypothesized that the magnitude and the direction of causal information flow between central and autonomic systems would be correlated with the trade-off between LTM and WM.

The physiological results across one night of sleep in Experiment 2 were consistent with those from Experiment 1 (Supplemental Figure S2, Table S5, S7). We confirmed that zolpidem increased sigma activity during sleep while suppressing vagal tone, measured by RMSSD and HF, but had no impact on LF. Similarly, we replicated the effective connectivity results (Figure $3 c)$, in which zolpidem increased effective connectivity ratio $(p=.0265)$, indicating greater causal influence of central sigma activity on autonomic vagal activity. 
We further assessed the functional roles of each physiological measure (EEG sigma activity, cardiac vagal activity, and effective connectivity ratio) on LTM and WM changes across sleep. We hypothesized that increasing sigma activity would benefit LTM retention in a wordpair-associates (WPA) task, whereas decreasing vagal activity would hinder WM improvement on a working memory operation span (OS) task. To this end, we examined overnight and 24-hr change scores in each task between the two drug conditions. For the word-pair task, our analysis showed that zolpidem significantly increased 24-hr LTM retention (Figure 4a right panel) and overnight retention (Figure 4a left panel). For the working memory operation span task, our analysis demonstrated that zolpidem decreased overnight improvement (Figure $4 \mathrm{~b}$ left panel) and 24-hr improvement (Figure $4 \mathrm{~b}$ right panel), compared to placebo. In summary, we confirmed our behavioral hypothesis that sigma-guided vagal suppression would increase LTM (Figure 4a) and decrease WM improvement (Figure 4b).

Next, we tested the correlations between each physiological measure (EEG sigma activity, cardiac vagal activity, and effective connectivity ratio) and memory changes across sleep using Pearson's correlation coefficients. We found a functional dissociation in vagal activity and behavior, where vagal activity during SWS was negatively correlated with LTM in the zolpidem condition (24-hr retention and HFln: $\mathrm{r}=-.460 ; \mathrm{p}=.018$; Figure $4 \mathrm{c}$ right panel), and positively correlated with WM improvement (overnight retention and HFln: $r=.422 ; \mathrm{p}=.032$; Figure $4 \mathrm{c}$ left panel) in the placebo condition. Correlational statistics between vagally-mediated HRV parameters and behavioral improvements are shown in Supplemental Table S9. No significant correlations were found between EEG sigma activity and WM improvement (zolpidem: all ps > .5687; placebo: all ps > .1943) or between EEG sigma activity and LTM retention (zolpidem: all ps > .15516; placebo: all ps > .1383). Taken together, vagal activity was positively associated with WM improvement, but inversely related to LTM.

We, then, asked whether central and autonomic antagonism impacted the trade-off between LTM and WM improvement by correlating the effective connectivity ratio with the normalized LTM-WM difference score, where higher numbers represent greater LTM than WM improvement. We found a positive correlation between the effective connectivity ratio and normalized LTM-WM difference score in the zolpidem $(\mathrm{r}=.429 ; \mathrm{p}=.020$; Figure $4 \mathrm{~d}$ right panel) and non-significant positive correlation in the placebo condition $(r=.251 ; \mathrm{p}=.190$; Figure $4 \mathrm{~d}$ left panel). These results suggested that the more central activity exerted influence on autonomic vagal activity, the more sleep was biased towards sigma-dependent LTM consolidation (and away from vagal-dependent WM processing).

Given the critical role of temporal coupling between sigma and SOs coupling for system consolidation, the current results lead to the prediction that sigma coupled with SOs supports LTM by suppressing WM processing. We tested this prediction by computing sigma power during the up-state of SOs and correlating this magnitude with the normalized LTM-WM difference score in the placebo condition. We found a significant positive correlation (Figure 5; Supplemental Table S10), suggesting evidence of competitive dynamics between the fundamental mechanisms of systems consolidation and WM processing.

\section{$\underline{\text { Discussion }}$}

The current work identified two neural mechanisms during NREM sleep that support the distinct enhancements in long-term and working memory. In study 1, we exploited the hypnotic zolpidem to enhance sigma activity during NREM sleep and report the novel finding that increasing sigma activity resulted in targeted vagal suppression during NREM. Next, we used the 
effective connectivity estimation technique to computationally test the causal hypothesis that central sigma activity actively suppressed vagal autonomic activity. Consistent with our hypothesis, results showed that central sigma exerted greater causal control over autonomic vagal activity and that pharmacologically increasing sigma activity boosted causal information flow from central to autonomic channels and decreased flow from autonomic to central channels. In a separate set of subjects, we replicated the pharmacological intervention and tested the functional significance of the central-autonomic mutual antagonism during NREM sleep by testing LTM and WM before and after a night of sleep. The physiological and effective connectivity results replicated those of experiment 1 . Moreover, the sigma-guided vagal suppression was associated with enhanced LTM retention at the cost of reduced WM improvement. Additionally, the magnitude of vagal suppression predicted a not previously reported trade-off between LTM and WM processing. These findings suggest evidence for a sleep switch that toggles between separate and non-overlapping NREM mechanisms that support LTM and WM processing. Furthermore, this switch can be biased towards greater LTM consolidation by boosting sigma activity.

Sigma activity is proposed to facilitate plasticity by producing long-term changes in responsiveness in cortical neurons [20] and increasing dendritic $\mathrm{Ca}^{2+}$ influxes [21], particularly enhanced when coupled to down-to-up transitions of the sleep slow oscillation [9]. Recently, Dickey and colleagues demonstrated the first evidence in humans that sigma activity may promote spike-timing-dependent plasticity (STDP), where correlated pre- and post-synaptic spiking within $25 \mathrm{~ms}$ windows modulated synaptic strength. STDP within this short window facilitates long-term potentiation (LTP), the cellular mechanism thought to underlie learning and memory [22]. Sigma activity, therefore, is thought to promote LTM via cortical synaptic plasticity. Furthermore, at the systems level, hippocampal-dependent long-term memory consolidation is supported by the replay of memory traces via triple phase coupling of hippocampal sharp-wave ripples nested within thalamic spindles and cortical SOs [23]. Several different interventions have demonstrated the causal role of sigma and spindle activity on hippocampal-dependent memory consolidation, including pharmacology [10] and targeted memory reactivation $[24,25]$. The current findings demonstrate that in addition to enhancing hippocampal-dependent memories, sigma activity, especially when coupled with SOs also suppresses subcortical vagal activity with significant functional outcomes, specifically a reduction in WM.

Vagal influence on cognitive function is a core principle of the Neurovisceral Integration Model [12], which posits that ANS activity is a peripheral index of the integrity of prefrontalautonomic networks that support inhibitory, goal-directed, high-order brain functions. The tenth cranial vagus nerve communicates peripheral information to and from the brainstem, with afferents projecting to higher-order, cognitive areas such as prefrontal cortex, anterior cingulate, and amygdala. Additionally, descending projections from the PFC to the brainstem and hypothalamic structures allow for bi-directional communication between the central nervous system and the ANS through the vagus nerve [12]. As such, high levels of vagally-mediated HRV is associated with superior executive function [26], working memory [13], and emotional regulation [27]. Prior cognitive training interventions revealed a link between autonomic vagal activity and improvements in working memory and other executive functions, suggesting that vagal activity might reflect enhanced cognitive control via greater automaticity and reduced activation between the striatum and prefrontal networks [28]. Although sleep is not typically measured across the cognitive training interventions, the current findings suggest that there may 
be a strengthening of prefrontal-autonomic networks during sleep that supports performance improvement.

Parasympathetic vagal activity is highest during SWS compared to all other states of consciousness [29]. Vagal activity is strongly coupled with low frequency, delta activity during SWS and vagal enhancement precedes the onset of SWS [30]. Several studies have linked SOs with WM improvement. For example, studies have shown that fronto-parietal SOs, but not sigma, predicts WM improvement [31,32]. However, not all studies report a consistent association between SOs and WM [33-35], and few accounts for autonomic activity. Chen and colleagues reported that vagal activity during SWS was a better predictor of WM improvement than SWA or vagal activity during wake [15]. In the current work, we found that changes in vagal autonomic activity during SWS, but not SOs per se, was critical for WM performance improvement. This, together with prior findings, suggests a non-negligible role of vagal influence on WM plasticity.

Given that both LTM and WM appear to rely on NREM sleep, one clear question emerges. Specifically, how are the limited resources of NREM sleep shared across memory processes? One answer may be mutual antagonistism - competing neural dynamics during NREM sleep support LTM or WM. In support of this theory, vagal nerve stimulation activates neurons in the locus coeruleus (LC) and increases NE levels in the brain [36, 37], and inactivation of LC impairs WM acquisition, while having no effect on consolidation or retention of spatial memories [38-42], whereas upregulating GABAergic networks impaired WM performance [43]. On the other hand, using ripple-triggered fMRI in monkeys, Logothetis and colleagues demonstrated that ripples orchestrate a privileged state of enhanced central brain activity by silencing output from the diencephalon, midbrain and brainstem, regions associated with autonomic regulation, which may serve to boost communication between hippocampus and cortex [16]. In addition, in both humans and mice, Lecci et al. (2017) demonstrated that heart rate and sigma power oscillate in antiphase with each other at $0.02 \mathrm{~Hz}$, suggesting a periodic switch between sigma and autonomic activation every 50 seconds [44].

Here, using effective connectivity, we demonstrated that a GABAergic agonist enhanced naturally occurring cortical sigma dominance over vagal autonomic activity. Similar vagolytic findings have been shown with zolpidem in persistent vegetative state patients [45]. Furthermore, the magnitude of this central influence predicted the trade-off between overnight LTM and WM improvement. Together with the previous literature, these finding suggest that sigma-dependent processes, including GABAergic hippocampal-thalamocortical networks, and vagal-dependent processes, including noradrenergic frontal-autonomic networks, may compete for sleep resources during NREM sleep. We hypothesize that the shared resource may be the SOs, which when coupled with sigma oscillations that carry ripples, promotes LTM and suppress other processes including WM; when not coupled with sigma, facilitate WM via enhancing prefrontal connection with other subcortical regions. We further hypothesize that sigma may act as a gating mechanism that block frontal-autonomic network and the resources of SOs for other processes, including WM, which might explain the mixed findings in the relation between SOs and WM. Given that approximately $20 \%$ of slow oscillations during NREM are sigma-coupled [46], this leaves plenty of resources to be divided amongst other processes, including WM. 
These data suggest a trade-off in which the two memory processes (LTM and WM) alternate during NREM sleep via a complex interaction at the synaptic (GABA vs NE activation), systems (thalamocortical vs frontal-midbrain), and mechanistic level (sigma-coupled SO vs uncoupled SO) (see graphical model in Figure 6). Further research is required to tease apart these mechanisms and test their generalizability across multiple cognitive domains and tasks. The sleep switch mechanism and separable sleep features associated with WM and LTM processing suggest directions for future translational research on cognitive disturbances observed in fronto-hippocampal neurodegenerative disorders such as Alzheimer's disease and in frontostriatal neurodegenerative disorders such as Parkinson's disease, both of which involve the decline of sleep $[47,48]$.

Acknowledgments: We thank undergraduate research assistants in the laboratory for assistance with data collection.

\section{Funding:}

National Institutes of Health R01AG046646 (S.C.M.)

Office of Naval Research, Young Investigator Award N00014-14-1-0513 (S.C.M.)

\section{Author contributions:}

Conceptualization: S.C.M., P.C., and L.N.W.

Methodology: P.C., H.N., and L.N.W.

Investigation: S.C.M., P.C., H.N., and L.N.W.

Visualization: P.C. and W.A.A.

Project administration: L.N.W.

Supervision: S.C.M.

Writing - original draft: P.C. and H.N.

Writing - review \& editing: S.C.M., P.C., and L.N.W.

Competing interests: Authors declare that they have no competing interests.

\section{Supplementary Materials}

Materials and Methods

Supplementary Text

Figs. S1 to S\#

Tables S1 to S\#

References (\#\#-\#\#) 
Fig. 1. Experimental design and behavioral tasks.

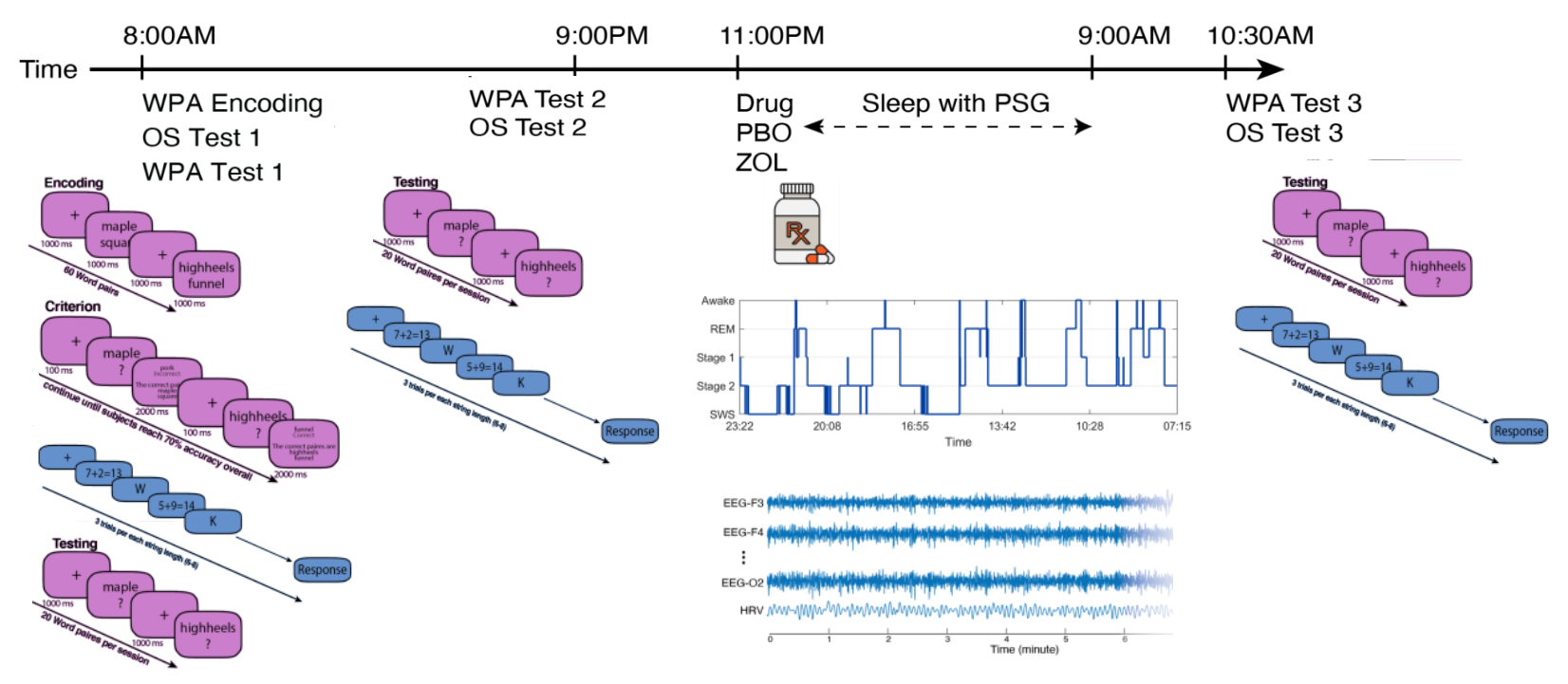

Experiment 1: Participants reported to the lab at 9:00PM and were hooked up to polysomnography (PSG), including electroencephalography (EEG), electrocardiogram (ECG), electromyogram (EMG), and electrooculogram (EOG). Before sleep, we recorded 5-min resting HRV while subjects lay awake in a still, supine position. At 11:00PM, directly before lights-out, subjects ingested either 10mg of zolpidem or placebo. Sleep was monitored online by a trained sleep technician. Participants were woken up at 9:00AM the next morning and permitted to leave the lab. Each participant experienced two visits per drug condition (a total of four visits).

Experiment 2: At 8:00AM, participants began encoding for the episodic memory word-pairedassociates (WPA) task, followed by the working memory operation-span task (OS) task and immediate recall for the WPA (Test 1). Participants left the lab after cognitive testing.

Participants were asked not to nap, exercise, or consume caffeine or alcohol, and were monitored with actigraphy during the break. Participants returned to the laboratory at 9:00 PM to complete the delayed recall over wake for WPA and OS (Test 2). Participants were then hooked up to polysomnography (PSG), including electroencephalography (EEG), electrocardiogram (ECG), electromyogram (EMG), and electrooculogram (EOG). Before sleep, we recorded 5-min resting HRV while subjects lay awake in a still, supine position. At 11:00PM, directly before lights-out, subjects ingested either 10mg of zolpidem or placebo. Sleep was monitored online by a trained sleep technician. Participants were woken up at 9:00AM the next morning and provided a standardized breakfast. At 10:30 AM, participants completed the delayed recall over sleep for WPA and OS (Test 3). For both tasks, to assess the change in performance, we measured two difference scores: overnight change (Test 3 - Test 2); 24-hr change (Test 3 - Test 1). Each participant experienced one visit per drug condition (a total of two visits). See Supplemental Figure S1 and Table S8 for summary statistics.

Word-paired associates (WPA) task: Subjects were visually presented with unrelated word pairs (e.g. maple-square) to maximize novel associations. Words were 3-9 letters in length and drawn from a normative set of English nouns. The WPA task consisted of an encoding phase and three recall phases. Encoding consisted viewing 60 pairs of words, each presented vertically 
stacked and shown twice in random order. Every word pair was presented for $1000 \mathrm{~ms}$ followed by a fixation cross for $1000 \mathrm{~ms}$. Immediate after the encoding, subjects are trained to criterion using a test in which participants were shown one word of the pair and were required to type in the associated word. Feedback was provided and participants had to achieve $70 \%$ accuracy to finish the training. Incorrect trials were repeated after a variable interval. Recall tests were assessed at immediate recall (9AM, Test 1), over wake (9PM, Test 2), and over sleep (10:30AM, Test 3). For testing, the 60 word-pairs were divided into three sets of 20 pairs, with one set tested at each test session and the order counterbalanced. To ensure that the subjects were not exposed to repeated stimulus, different versions of word lists for each visit were imposed.

Operation Span (OS) task: Participants performed mental arithmetic while memorizing a set of unrelated letters. The task included 3 practice and 9 test trials. Participants were tested in letter strings six to eight. For each letter string, participants were shown a series of math problems that they had to confirm were correct within 3 seconds, using pre-determined responses on the keyboard. After each equation, a letter would appear on the screen and the subject was instructed to remember each letter. At the end of each string, the participant was instructed to recall the letters in the order presented by typing responses on a computer keyboard. Immediately after each trial, the next letter string would be presented. An example of a six-item trial might be: 12 $2=8($ correct/ incorrect? $)=>\mathrm{J} ; 6+7=14$ (correct/incorrect?) $=>\mathrm{G} ; 3-2=1$

$($ correct/incorrect? $)=>\mathrm{S} ; 5+7=13($ correct/incorrect? $)=>\mathrm{K} ; 8+7=14($ correct/incorrect? $)=>$ $\mathrm{H} ; 5+9=14$ (correct/incorrect?) $=>\mathrm{L}$. After verifying the six equations in this example, participants were asked to type the presented letters in the order that they were presented (in this case JGSKHL). If the participants forgot one of the letters in a trial, they were instructed to provide their best guess. In addition, to decrease trade-off between solving the operations and remembering the letters, an $85 \%$ accuracy criterion on the math operations was required for all the participants. Based on this criterion, four participants during the ZOL condition were excluded. We calculated performance as: number of correct letters recalled in the correct position divided by total number of letters in the string per trial, and then averaged over the total trials. 
Fig. 2. Zolpidem decreased vagally-mediated HRV, but not LF, during SWS.
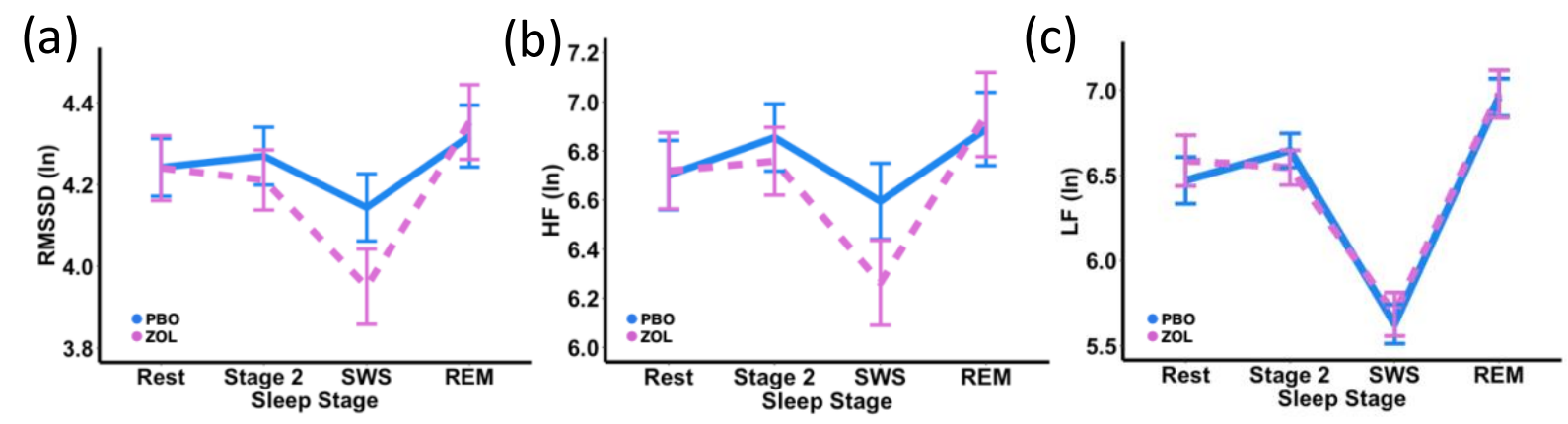

(a) For RMSSD, we report a significant main effect of sleep stage $(\mathrm{F}(3,366)=21.257$, $\mathrm{p}<$ .0001 ), with a decreased HRV during SWS compared to Rest, Stage 2, and REM (all ps $<.0001$ ). We also found a significant interaction $(F(3,366)=3.8630, p=.0096)$ between sleep stage and drug condition, with decreased vagal activity during SWS $(\mathrm{p}=.0006)$ in zolpidem compared with placebo, but not during Stage $2(\mathrm{p}=.3549)$, REM $(\mathrm{p}=.3804)$, or Rest $(\mathrm{p}=.6152)$. The likelihood ratio test was significant $(\mathrm{LR}=13.8544 ; \mathrm{p}=.0078)$, suggesting that zolpidem significantly modulated the time-domain measure of HRV.

(b) For HF, we report a significant main effect of sleep stage $(F(3,366)=16.9891, p<.0001)$, with a decreased HRV during SWS compared to Rest $(\mathrm{p}=.0006)$, Stage $2(\mathrm{p}<.0001)$, and REM $(\mathrm{p}<.0001)$. Similarly, we also report a significant interaction $(\mathrm{F}(3,366)=3.1899, \mathrm{p}=.0238)$ between sleep stage and drug condition, with decreased vagal activity during SWS $(p=.0020)$ in zolpidem compared with placebo, but not during Stage $2(p=.4194)$, REM $(p=.4365)$, or Rest $(p=.6070)$. The likelihood ratio test was significant $(L R=11.3671 ; p=.0227)$, suggesting that zolpidem significantly modulated the frequency-domain measure of HRV.

(c) For LF, we report a significant main effect of sleep stage $(\mathrm{F}(3,366)=93.0330, \mathrm{p}<.0001)$, with a decreased LF power during SWS compared to Rest, Stage 2, and REM (all ps $<.0001$ ), and an increased LF power during REM compared to Rest and Stage 2 (all ps $<.0001$. No significant main effect of drug condition $(p=.6337)$, nor interaction between sleep stage and drug condition $(\mathrm{p}=.5681)$ were found. The likelihood ratio test was not significant $(\mathrm{LR}=$ 2.2889; $\mathrm{p}=.6828)$, suggesting that zolpidem did not significantly modulate low frequency HRV. 
Fig. 3. Effective Connectivity Modulated by Drug Condition.

(a)

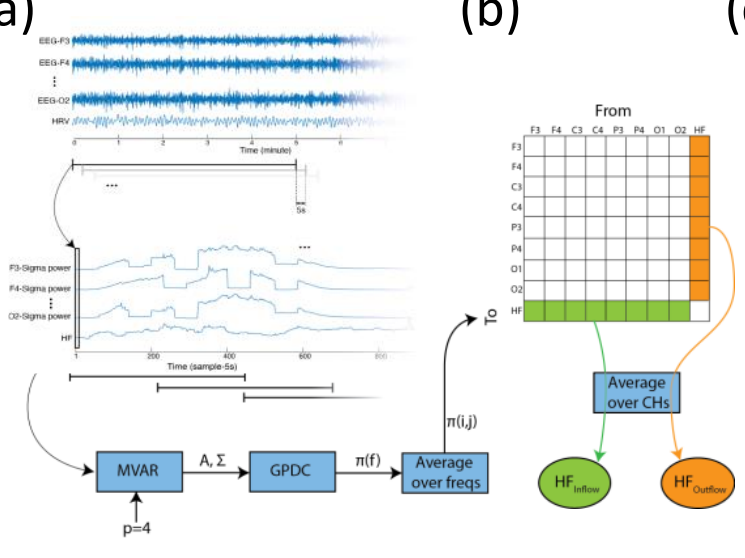

(c)

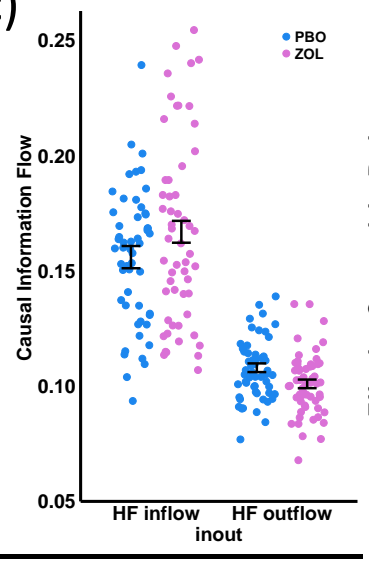

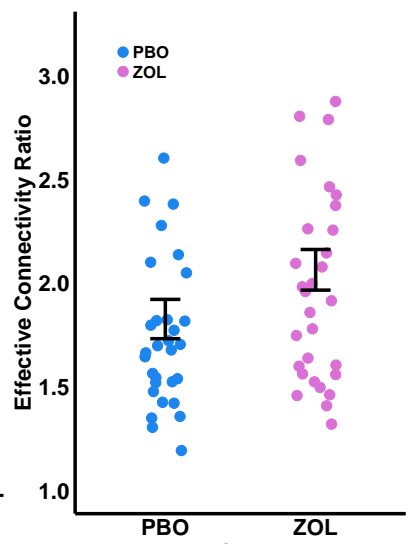

(a) Effective Connectivity Estimation Procedure (see Supplemental Methods)

(b) Experiment 1: We report a main effect of inflow vs outflow $(\mathrm{F}(1,185)=273.317, \mathrm{p}<.0001)$, with a greater HFinflow than HFoutflow in both drug conditions; an interaction between drug condition and inflow vs outflow $(\mathrm{F}(1,185)=5.744, \mathrm{p}=.0175)$, with a greater HFinlfow during zolpidem compared to placebo $(\mathrm{p}=.0369)$. No main effect of drug condition was found $(\mathrm{F}(1$, $185)=0.512, p=.4751)$. The likelihood ratio test was significant $(L R=6.0745 ; p=.0480)$, suggesting that zolpidem significantly modulated the causal information flow between sigma and HF activity. Effective connectivity ratios (HFInflow/ HFOutflow) increased significantly during the zolpidem night $(\mathrm{F}(1,79)=8.0607, \mathrm{p}=.0059)$.

(c) Experiment 2: Effective connectivity ratios (HFInflow/ HFOutflow) increased significantly during the zolpidem night $(\mathrm{F}(1,32)=5.4087, \mathrm{p}=.0265)$. 
Fig. 4. Zolpidem increases LTM, but decreases WM improvement.

(a)

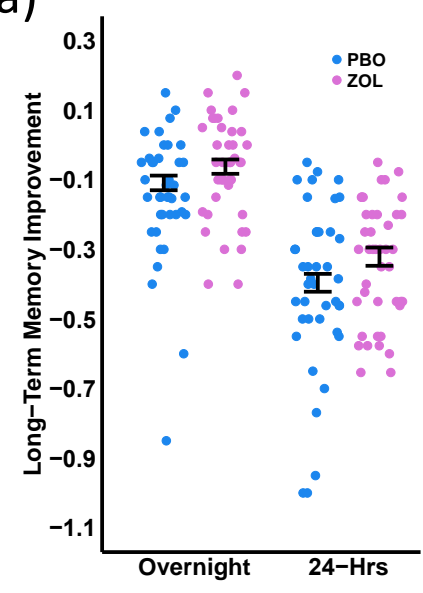

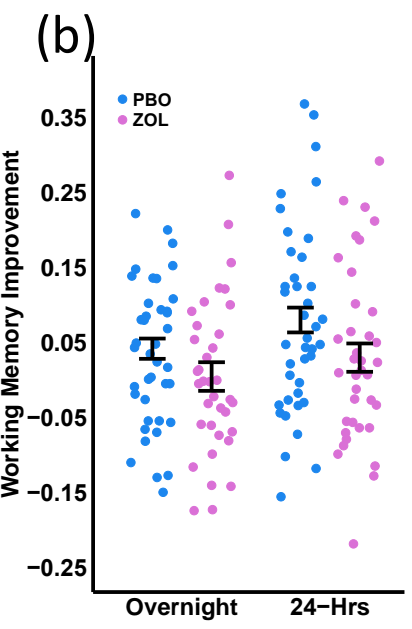

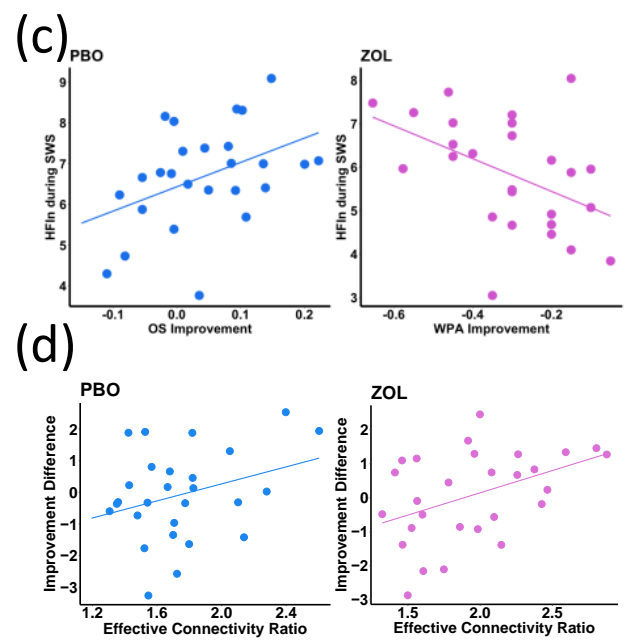

(a) Long-term memory (WPA task) improvement by drug conditions and time. (Y axis: WPA Overnight [Test3-Test2] and 24-hr [Test3-Test1] improvement; asterisks indicate significant differences in behavioral changes between two drug conditions; $\left.{ }^{*} \mathrm{p}<0.05\right)$ ZOL yielded greater but not significant overnight retention of WPA than the PBO visits (estimate $=-0.1156, \mathrm{CI}=(-0.2408$, $-0.0095), \mathrm{t}=-1.8104, \mathrm{p}=0.0810)$, accounting for visit, as well as greater 24-hr retention of WPA than $\mathrm{PBO}$ visits $($ estimate $=-0.1810, \mathrm{CI}=(-0.3519,-0.0096), \mathrm{t}=-2.0704, \mathrm{p}=0.0474)$, accounting for visit.

(b) Working memory (OS task) improvement by drug conditions and time. (Y axis: OS Overnight [Test3-Test2] and 24-hr [Test3-Test1] improvement; asterisks indicate significant differences in behavioral changes between two drug conditions; *p<0.05) PBO showed significantly greater overnight improvement of OS than ZOL visits (estimate $=0.1242, \mathrm{CI}=$ (0.0201, 0.2284), $\mathrm{t}=2.3377, \mathrm{p}=0.0260)$, accounting for Test 2 performance and visit, as well as greater but not significant $24-\mathrm{hr}$ improvement of OS than ZOL visits (estimate $=0.1000, \mathrm{CI}=(-$ $0.0184,0.2185), \mathrm{t}=1.6546, \mathrm{p}=0.1081$ ), accounting for Test 1 performance and visit.

(c) Functional role of vagal activity on memory. ( $\mathrm{Y}$ axis: HFln during SWS, $\mathrm{X}$ axis: OS overnight and WPA 24-hr improvement) Vagal activity during SWS positively predicted working memory (OS task) improvement $(\mathrm{r}=.422 ; \mathrm{p}=.032)$ but negatively predicted long-term memory (WPA task) improvement $(\mathrm{r}=-.460 ; \mathrm{p}=.018)$.

(d) Functional role of effective connectivity ratio on memory trade-off. ( $\mathrm{Y}$ axis: normalized WPA improvement - normalized OS improvement score, $\mathrm{X}$ axis: effective connectivity ratio = HFInflow/ HFOutflow) Effective connectivity ratio (a higher ratio indicates a greater causal effect from sigma to vagal) during sleep positively predicted memory trade-off (a greater difference indicates a greater improvement in the WPA task than the OS task) during the zolpidem night $(\mathrm{r}=$ $.429 ; \mathrm{p}=.020)$, but not the placebo night $(\mathrm{r}=.251 ; \mathrm{p}=.190)$. 
Fig. 5. Functional roles of sigma power coupled with SO up-state on LTM and WM.
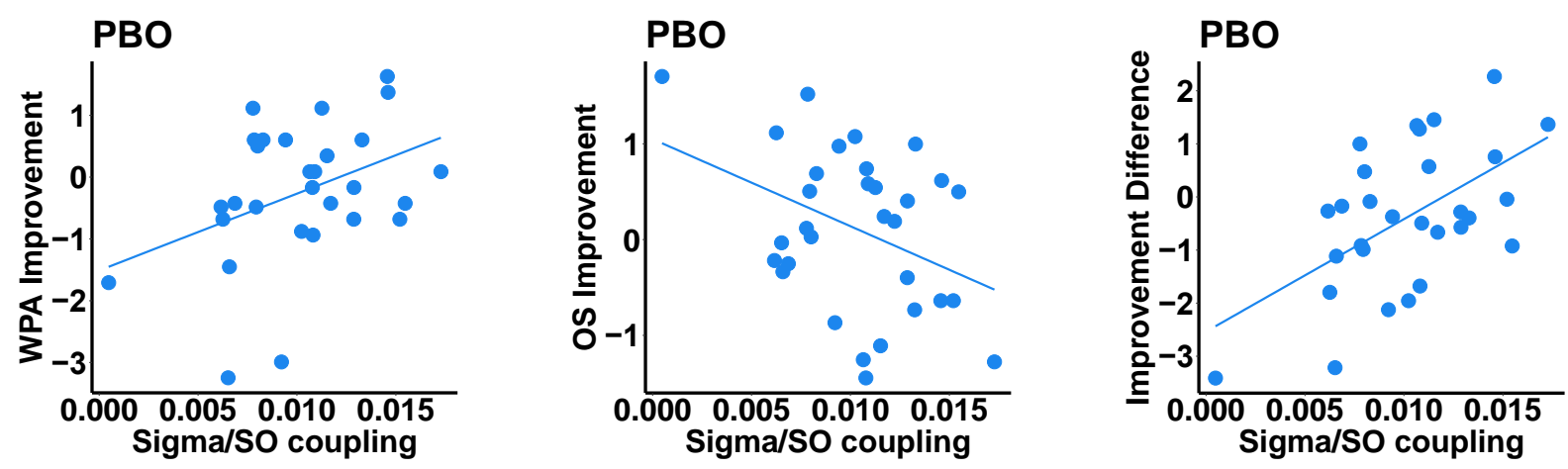

(a) Long-term memory (WPA task) improvement positively correlated with sigma power coupled with SO up-state. (Y axis: normalized score of WPA 24-hr improvement; X axis: normalized sigma power coupled during the up-state of SOs; $r=.400 ; p=.034$ )

(b) Working memory (OS task) improvement negatively correlated with sigma power coupled with SO up-state. (Y axis: normalized score of OS overnight improvement; X axis: normalized sigma power coupled during the up-state of SOs; $r=-.380 ; p=.033$ )

(C) Improvement difference positively correlated with sigma power coupled with SO upstate. (Y axis: normalized WPA improvement - normalized OS improvement score; X axis: normalized sigma power coupled during the up-state of SOs; $r=.560 ; p=.002$ ) 
Fig. 6. Sleep Switch Model

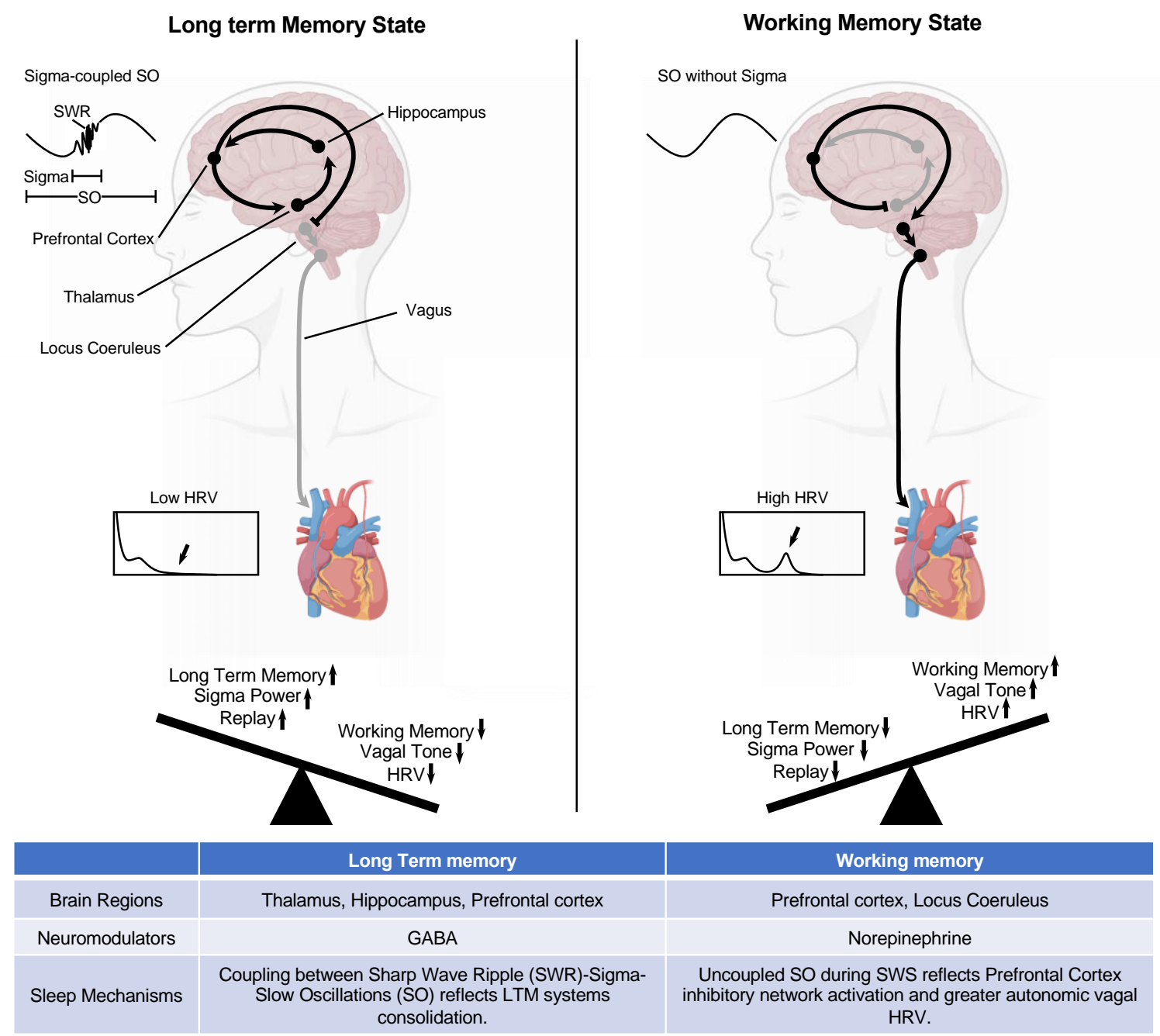

The model represents the proposed brain regions, primary neuromodulators, and sleep mechanisms involved in the Long-term memory state and the Working memory state that toggle throughout non-rapid eye movement (NREM) sleep. During the Long-Term Memory state, consolidation occurs via sigma-coupled SOs, which leads to reduced autonomic vagal-dependent activity and less WM improvement. During the Working Memory state, greater efficiency occurs during uncoupled SOs associated with increased autonomic vagal-dependent activity, which leads to reduced central sigma-dependent activity and less LTM consolidation. 


\section{References}

1. James, W. (1890). The principles of psychology, Henry Holt and Co. https://doi.org/10.1037/10538-000

2. Funahashi, S., Bruce, C. J., \& Goldman-Rakic, P. S. (1989). Mnemonic coding of visual space in the monkey's dorsolateral prefrontal cortex. Journal of Neurophysiology, 61(2), 331-349.

3. Squire, L. R., \& Zola-Morgan, S. (1991). The medial temporal lobe memory system. Science, 253(5026), 1380-1386.

4. Hoskin, A. N., Bornstein, A. M., Norman, K. A., \& Cohen, J. D. (2019). Refresh my memory: Episodic memory reinstatements intrude on working memory maintenance. Cognitive, Affective, \& Behavioral Neuroscience, 19(2), 338-354.

5. D'Esposito, M., \& Grafman, J. H. (Eds.). (2019). Handbook of Clinical Neurology 3rd Series. In Handbook of Clinical Neurology (Vol. 163, p. v). Elsevier. https://doi.org/10.1016/B978-0-12-804281-6.09993-6

6. Ranganath, C., \& Blumenfeld, R. S. (2005). Doubts about double dissociations between short-and long-term memory. Trends in Cognitive Sciences, 9(8), 374-380.

7. Rasch, B., \& Born, J. (2013). About sleep's role in memory. Physiological Reviews, 93(2), 681-766. PubMed. https://doi.org/10.1152/physrev.00032.2012

8. Ngo, H.-V., Fell, J., \& Staresina, B. (2020). Sleep spindles mediate hippocampal-neocortical coupling during long-duration ripples. Elife, 9, e57011.

9. Niethard, N., Ngo, H.-V. V., Ehrlich, I., \& Born, J. (2018). Cortical circuit activity underlying sleep slow oscillations and spindles. Proceedings of the National Academy of Sciences, 115(39), E9220-E9229.

10. Mednick, S. C., McDevitt, E. A., Walsh, J. K., Wamsley, E., Paulus, M., Kanady, J. C., \& Drummond, S. P. A. (2013). The Critical Role of Sleep Spindles in Hippocampal-Dependent Memory: A Pharmacology Study. The Journal of Neuroscience, 33(10), 4494. https://doi.org/10.1523/JNEUROSCI.3127-12.2013

11. Compte, A., Brunel, N., Goldman-Rakic, P. S., \& Wang, X.-J. (2000). Synaptic mechanisms and network dynamics underlying spatial working memory in a cortical network model. Cerebral Cortex, 10(9), 910-923.

12. Thayer, J. F., Hansen, A. L., Saus-Rose, E., \& Johnsen, B. H. (2009). Heart Rate Variability, Prefrontal Neural Function, and Cognitive Performance: The Neurovisceral Integration Perspective on Self-regulation, Adaptation, and Health. Annals of Behavioral Medicine, 37(2), 141-153. https://doi.org/10.1007/s12160-009-9101-z

13. Mosley, E., Laborde, S., \& Kavanagh, E. (2018). Coping related variables, cardiac vagal activity and working memory performance under pressure. Acta Psychologica, 191, 179-189. https://doi.org/10.1016/j.actpsy.2018.09.007

14. Zinke, K., Noack, H., \& Born, J. (2018). Sleep augments training-induced improvement in working memory in children and adults. Neurobiology of Learning and Memory, 147, 46-53. https://doi.org/10.1016/j.nlm.2017.11.009

15. Chen, P.-C., Whitehurst, L. N., Naji, M., \& Mednick, S. C. (2020). Autonomic Activity during a Daytime Nap Facilitates Working Memory Improvement. Journal of Cognitive Neuroscience, 1-12. https://doi.org/10.1162/jocn_a_01588 
16. Logothetis, N. K., Eschenko, O., Murayama, Y., Augath, M., Steudel, T., Evrard, H., Besserve, M., \& Oeltermann, A. (2012). Hippocampal-cortical interaction during periods of subcortical silence. Nature, 491(7425), 547-553.

17. Novitskaya, Y., Sara, S. J., Logothetis, N. K., \& Eschenko, O. (2016). Ripple-triggered stimulation of the locus coeruleus during post-learning sleep disrupts ripple/spindle coupling and impairs memory consolidation. Learning \& Memory, 23(5), 238-248.

18. Friston, K. J. (2011). Functional and Effective Connectivity: A Review. Brain Connectivity, 1(1), 13-36. https://doi.org/10.1089/brain.2011.0008

19. Drover, D. R. (2004). Comparative pharmacokinetics and pharmacodynamics of short-acting hypnosedatives: Zaleplon, zolpidem and zopiclone. Clinical Pharmacokinetics, 43(4), 227238. https://doi.org/10.2165/00003088-200443040-00002

20. Timofeev, I., Grenier, F., Bazhenov, M., Houweling, A. R., Sejnowski, T. J., \& Steriade, M. (2002). Short-and medium-term plasticity associated with augmenting responses in cortical slabs and spindles in intact cortex of cats in vivo. The Journal of Physiology, 542(2), 583598.

21. Seibt, J., Richard, C. J., Sigl-Glöckner, J., Takahashi, N., Kaplan, D. I., Doron, G., de Limoges, D., Bocklisch, C., \& Larkum, M. E. (2017). Cortical dendritic activity correlates with spindle-rich oscillations during sleep in rodents. Nature Communications, 8(1), 1-13.

22. Dickey, C. W., Sargsyan, A., Madsen, J. R., Eskandar, E. N., Cash, S. S., \& Halgren, E. (2021). Travelling spindles create necessary conditions for spike-timing-dependent plasticity in humans. Nature Communications, 12(1), 1027. https://doi.org/10.1038/s41467-021-21298$\underline{\mathrm{X}}$

23. Latchoumane, C.-F. V., Ngo, H.-V. V., Born, J., \& Shin, H.-S. (2017). Thalamic Spindles Promote Memory Formation during Sleep through Triple Phase-Locking of Cortical, Thalamic, and Hippocampal Rhythms. Neuron, 95(2), 424-435.e6. https://doi.org/10.1016/j.neuron.2017.06.025

24. Antony, J. W., Piloto, L., Wang, M., Pacheco, P., Norman, K. A., \& Paller, K. A. (2018). Sleep Spindle Refractoriness Segregates Periods of Memory Reactivation. Current Biology, 28(11), 1736-1743.e4. https://doi.org/10.1016/j.cub.2018.04.020

25. Cairney, S. A., Guttesen, A. á V., El Marj, N., \& Staresina, B. P. (2018). Memory Consolidation Is Linked to Spindle-Mediated Information Processing during Sleep. Current Biology, 28(6), 948-954.e4. https://doi.org/10.1016/j.cub.2018.01.087

26. Williams, P. G., Cribbet, M. R., Tinajero, R., Rau, H. K., Thayer, J. F., \& Suchy, Y. (2019). The association between individual differences in executive functioning and resting highfrequency heart rate variability. Biological Psychology, 148, 107772. https://doi.org/10.1016/j.biopsycho.2019.107772

27. Mather, M., \& Thayer, J. F. (2018). How heart rate variability affects emotion regulation brain networks. Current Opinion in Behavioral Sciences, 19, 98-104.

28. Lin, F., L Heffner, K., Ren, P., \& Tadin, D. (2017). A role of the parasympathetic nervous system in cognitive training. Current Alzheimer Research, 14(7), 784-789.

29. Whitehurst, L. N., Chen, P.-C., Naji, M., \& Mednick, S. C. (2020). New directions in sleep and memory research: The role of autonomic activity. Current Opinion in Behavioral Sciences, 33, 17-24. https://doi.org/10.1016/j.cobeha.2019.11.001

30. Rothenberger, S. D., Krafty, R. T., Taylor, B. J., Cribbet, M. R., Thayer, J. F., Buysse, D. J., Kravitz, H. M., Buysse, E. D., \& Hall, M. H. (2015). Time-varying correlations between 
delta EEG power and heart rate variability in midlife women: The SWAN Sleep Study. Psychophysiology, 52(4), 572-584. https://doi.org/10.1111/psyp.12383

31. Ferrarelli, F., Kaskie, R., Laxminarayan, S., Ramakrishnan, S., Reifman, J., \& Germain, A. (2019). An increase in sleep slow waves predicts better working memory performance in healthy individuals. NeuroImage, 191, 1-9. https://doi.org/10.1016/j.neuroimage.2019.02.020

32. Pugin, F., Metz, A. J., Wolf, M., Achermann, P., Jenni, O. G., \& Huber, R. (2015). Local increase of sleep slow wave activity after three weeks of working memory training in children and adolescents. Sleep, 38(4), 607-614. https://doi.org/10.5665/sleep.4580

33. Chen, P.-C., Whitehurst, L. N., Naji, M., \& Mednick, S. C. (2020). Autonomic/central coupling benefits working memory in healthy young adults. Neurobiology of Learning and Memory, 173, 107267. https://doi.org/10.1016/j.nlm.2020.107267

34. Lau, E. Y. Y., Wong, M. L., Lau, K. N. T., Hui, F. W. Y., \& Tseng, C. (2015). Rapid-EyeMovement-Sleep (REM) Associated Enhancement of Working Memory Performance after a Daytime Nap. PLOS ONE, 10(5), e0125752. https://doi.org/10.1371/journal.pone.0125752

35. MacDonald, K. J., Lockhart, H. A., Storace, A. C., Emrich, S. M., \& Cote, K. A. (2018). A daytime nap enhances visual working memory performance and alters event-related delay activity. Cognitive, Affective, \& Behavioral Neuroscience, 18(6), 1105-1120. https://doi.org/10.3758/s13415-018-0625-1

36. Hassert, D. L., Miyashita, T., \& Williams, C. L. (2004). The Effects of Peripheral Vagal Nerve Stimulation at a Memory-Modulating Intensity on Norepinephrine Output in the Basolateral Amygdala. Behavioral Neuroscience, 118(1), 79-88. https://doi.org/10.1037/0735-7044.118.1.79

37. Roosevelt, R. W., Smith, D. C., Clough, R. W., Jensen, R. A., \& Browning, R. A. (2006). Increased extracellular concentrations of norepinephrine in cortex and hippocampus following vagus nerve stimulation in the rat. Brain Research, 1119(1), 124-132. https://doi.org/10.1016/j.brainres.2006.08.048

38. Chamberlain, S. R., Müller, U., Blackwell, A. D., Robbins, T. W., \& Sahakian, B. J. (2006). Noradrenergic modulation of working memory and emotional memory in humans. Psychopharmacology, 188(4), 397-407. https://doi.org/10.1007/s00213-006-0391-6

39. Khakpour-Taleghani, B., Lashgari, R., Motamedi, F., \& Naghdi, N. (2009). Effect of reversible inactivation of locus ceruleus on spatial reference and working memory. Neuroscience, 158(4), 1284-1291.

40. Beste, C., Steenbergen, L., Sellaro, R., Grigoriadou, S., Zhang, R., Chmielewski, W., Stock, A.-K., \& Colzato, L. (2016). Effects of concomitant stimulation of the GABAergic and norepinephrine system on inhibitory control-a study using transcutaneous vagus nerve stimulation. Brain Stimulation, 9(6), 811-818.

41. Sun, L., Peräkylä, J., Holm, K., Haapasalo, J., Lehtimäki, K., Ogawa, K. H., Peltola, J., \& Hartikainen, K. M. (2017). Vagus nerve stimulation improves working memory performance. Journal of Clinical and Experimental Neuropsychology, 39(10), 954-964. https://doi.org/10.1080/13803395.2017.1285869

42. Pihlaja, M., Failla, L., Peräkylä, J., \& Hartikainen, K. M. (2020). Reduced Frontal Nogo-N2 With Uncompromised Response Inhibition During Transcutaneous Vagus Nerve Stimulation-More Efficient Cognitive Control? Frontiers in Human Neuroscience, 14, 391. https://doi.org/10.3389/fnhum.2020.561780 
43. Lozano-Soldevilla, D., ter Huurne, N., Cools, R., \& Jensen, O. (2014). GABAergic Modulation of Visual Gamma and Alpha Oscillations and Its Consequences for Working Memory Performance. Current Biology, 24(24), 2878-2887. https://doi.org/10.1016/j.cub.2014.10.017

44. Lecci, S., Fernandez, L. M. J., Weber, F. D., Cardis, R., Chatton, J.-Y., Born, J., \& Lüthi, A. (2017). Coordinated infraslow neural and cardiac oscillations mark fragility and offline periods in mammalian sleep. Science Advances, 3(2), e1602026. https://doi.org/10.1126/sciadv.1602026

45. Machado, Estevez, Rodriguez, Perez-Nellar, Chinchilla, Philip DeFina, Gerry Leisman, Frederick R. Carrick, Robert Melillo, Adam Schiavi, Joel Gutierrez, Maylen Carballo, Andres Machado, \& Ana Olivares and Nuvia Perez-Cruz. (2014). Zolpidem Arousing Effect in Persistent Vegetative State Patients: Autonomic, EEG and Behavioral Assessment. Current Pharmaceutical Design, 20(26), 4185-4202. https://doi.org/10.2174/13816128113196660646

46. Malerba, P., Whitehurst, L. N., Simons, S. B., \& Mednick, S. C. (2018). Spatio-temporal structure of sleep slow oscillations on the electrode manifold and its relation to spindles. Sleep, 42(zsy197). https://doi.org/10.1093/sleep/zsy197

47. Mander, B. A., Winer, J. R., \& Walker, M. P. (2017). Sleep and Human Aging. Neuron, 94(1), 19-36. PubMed. https://doi.org/10.1016/j.neuron.2017.02.004

48. Targa, A. D. S., Rodrigues, L. S., Noseda, A. C. D., Aurich, M. F., Andersen, M. L., Tufik, S., da Cunha, C., \& Lima, M. M. S. (2016). Unraveling a new circuitry for sleep regulation in Parkinson's disease. Neuropharmacology, 108, 161-171. https://doi.org/10.1016/j.neuropharm.2016.04.018

49. Dang-Vu, T. T., Schabus, M., Desseilles, M., Albouy, G., Boly, M., Darsaud, A., Gais, S., Rauchs, G., Sterpenich, V., Vandewalle, G., Carrier, J., Moonen, G., Balteau, E., Degueldre, C., Luxen, A., Phillips, C., \& Maquet, P. (2008). Spontaneous neural activity during human slow wave sleep. Proceedings of the National Academy of Sciences, 105(39), 15160. https://doi.org/10.1073/pnas.0801819105 